\title{
The Effect of Proactive Personality on the Thriving at Work Among New Military Civilian: The Mediating Effect of Perceived Organizational Support
}

\author{
Tian Yanhui, Du Keke, Bai Shengjun, Bi Junfei \\ College of Information and Communication, National University of Defense Technology, Xi'an, China
}

\section{Email address:}

tianyanhui05@163.com (Tian Yanhui),48059821@qq.com (Du Keke), 928869761@qq.com (Bai Shengjun),

1774997983@qq.com (Bi Junfei)

\section{To cite this article:}

Tian Yanhui, Du Keke, Bai Shengjun, Bi Junfei. The Effect of Proactive Personality on the Thriving at Work Among New Military Civilian: The Mediating Effect of Perceived Organizational Support. Science Innovation. Vol. 9, No. 2, 2021, pp. 36-41.

doi: 10.11648/j.si.20210902.11

Received: December 14, 2020; Accepted: March 26, 2021; Published: March 30, 2021

\begin{abstract}
To explore the influence factor and the internal mechanism of the thriving at work among new military civilian, a total of 513 military civilian were investigated by the proactive personality scale, the perceived organizational support scale and the thriving at work scale. The results of correlation analysis shows that, the proactive personality was significantly positively correlated with the thriving at work $(r=0.40, \mathrm{p}<0.01)$ and the organizational support $(\mathrm{r}=0.33, \mathrm{p}<0.01)$; the results also shows that the correlation between the perceived organizational support and the thriving at work was significant $(\mathrm{r}=0.52, \mathrm{p}<0.01)$. In order to examine the effect of the proactive personality on the thriving at work and the effect of the organizational support among military civilian, the results of structural equation model shows that, the proactive personality had a significant positive effect on the thriving at work $(\beta=0.60, p<0.001)$, and the perceived organizational support played a partly mediation effect between the proactive personality and the thriving at work, and the mediation size was 0.172 . This research reveals the influence factors of the thriving at work and the internal mechanism, and it also has some enlightening significance for the education, motivation and management of Military civilian, and it could draw the following conclusions, in order to enhance the thriving at work of military civilian, we can recruit or cultivate the proactive personality and provide necessary organizational support.
\end{abstract}

Keywords: New Military Civilian, Proactive Personality, Thriving at Work, Perceived Organizational Support

\section{军队新入职文职人员主动性人格对工作旺盛的影响：组织支持感 的中介作用}

田艳辉, 杜柯柯, 白胜军, 毕俊菲

国防科技大学信息通信学院, 西安, 中国

\section{邮箱}

tianyanhui05@163.com（田艳辉），48059821@qq.com（杜柯柯）, 928869761@qq.com（白胜军），1774997983.qq.com（毕俊菲）

摘要：为探讨军队新入职文职人员工作旺盛感的影响因素及内部作用机制，采用主动性人格量表、组织支持感量表和 工作旺盛感量表对 513 名军队文职人员进行调查。相关分析的结果显示, 主动性人格与工作旺盛感 $(r=0.40, p<0.01)$ 和组织支持感 $(r=0.33, p<0.01)$ 均显著正相关; 组织支持感与工作旺盛感间 $(r=0.52, p<0.01)$ 的相关也达到显著性 水平。在对军队文职人员主动性人格对工作旺盛感的影响及组织支持感在两者间的作用进行检验时, 结构方程模型的 结果显示，文职人员主动性人格对工作旺盛感具有显著的正向影响 $(\beta=0.60, p<0.001)$, 组织支持感在主动性人格影 
响工作旺盛感的关系中起部分中介作用, 中介效应大小为 0.172 。本研究揭示了影响文职人员工作旺盛感的因素及内部 作用机制, 对文职人员的教育、激励和管理也具有一定的启示意义, 提升文职人员的工作旺盛感可以从招聘或培养文 职人员的主动性人格以及提供必要的组织支持入手。

关键词: 新入职文职人员，主动性人格，工作旺盛感，组织支持感

\section{1. 引言}

随着军事人力资源政策制度的改革, 越来越多的地方 优秀青年进入军队文职人员队伍, 成为我军未来建设与发 展的生力军。公开报道显示, 2018年我军首次公开面向社 会招聘文职人员 9297 人， 2019 年招录 19523 人，2020年招 录 27073 人, 且有逐年增加的趋势, 可以预测, 文职人员 将会成为我军军事人力资源的重要组成部分, 在未来发挥 越来越重要的作用。因此, 对文职人员工作态度及行为的 相关变量进行研究, 探究其影响因素和作用机制, 对文职 人员的有效使用和管理具有重要的意义和作用。

工作旺盛感是随着积极心理学的兴起而逐渐进入 人们研究视野的工作状态, 指的是个体在工作中同时体 会到活力和学习的一种心理状态 [1], 作为个体积极发展 状态的重要标志之一, 工作旺盛感高的个体不仅具有更 强的职场适应能力 [2], 而且能够有效降低心理消耗 [3], 维持较高的心理健康水平 [4], 因此, 对工作旺盛感的研 究一直是积极组织行为学领域中重要的研究变量。对于 初入职的军队文职人员来说, 职业价值观和职业态度尚 未完全定型, 提高其工作旺盛感不仅有利于提高组织绩 效 [5], 而且对个人发展也大有益处。以往关于工作旺盛 感影响因素的研究中, 大致可以分为两类, 第一类为工 作情境, 如领导类型[6]、职场友谊[7]、人-情境交互作 用 [8]对工作旺盛感的影响; 第二类为工作资源, 如工作 家庭增益 [9]、组织社会化 [10]等因素对工作旺盛感的影 响, 而对于人格与工作旺盛感间关系的研究较为少见。 主动性人格又称为前瞻性人格或者前摄性人格, 指的是 能够对环境改变产生影响的稳定的人格特质[11], 主动 性人格的个体倾向于主动改变周围环境, 而不被周围情 境阻力所制约, 以此可以获得更强的控制感, 由此带来 更好的组织内及组织外绩效, 已有研究表明主动性人格 与职业成熟度、进谏行为、创新行为[12-14]等积极工作 行为有显著的正向相关作用。由此, 本研究预测, 文职 人员主动性人格可能会对工作旺盛感产生显著的促进 作用。

组织支持感是个体对组织如何看待他们贡献并关心 他们利益的一种看法和知觉, 简言之, 就是对个体感受到 的来自组织的支持 [15]。主动性人格的个体除了具有主动 改变环境的特点外, 还善于利用周边资源, 构建更为广泛 的朋友圈, 充分发掘和利用身边的资源为自身服务。同时, 作为一种被当下社会所推崇的一种人格特征, 主动性人格 的个体往往会受到更多领导及同事的赞扬和鼓励, 在工作 过程中也更容易获得支持, 这些工作场所的支持都会被当 事人知觉为组织对自己的厚爱, 由此感受到强烈的组织支 持感。而组织支持感对工作旺盛感的促进作用可以从工作
要求一资源模型中得以解释, 该理论认为, 任何工作都包 含工作需求和工作资源两个维度, 他们分别从能量损耗过 程和能力激励过程来影响个体的工作绩效, 过高的工作需 求降低工作绩效甚至引发个体的健康问题, 而充分的工作 资源, 既可以作为外在助力也可作为内部动力促使个体实 现更高的绩效 [16]。而组织支持感作为工作领域中的特定 工作资源, 势必会促进个体达成更高的工作目标和绩效, 以此带来强烈的工作满足感和旺盛感。基于以上分析, 本 研究提出如下研究假设: 文职人员主动性人格既可以直接 影响工作旺盛感，也可通过组织支持感的“桥梁”作用间接 影响工作旺盛感。研究模型如图1所示。

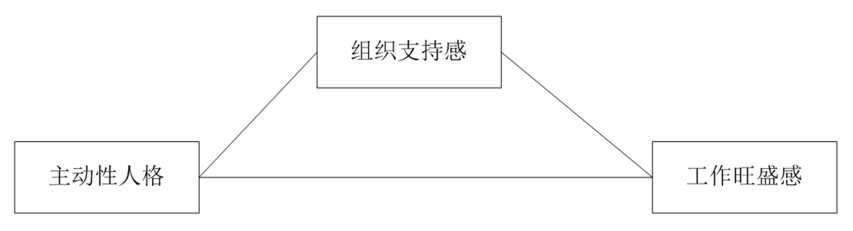

图1 研究模型图。

\section{2. 研究方法}

\section{1. 研究对象}

采用整群方便抽样的方法, 利用军队新入职文职人员 集中培训时机, 从陕西省西安市、湖北省武汉市和湖南省 长沙市等地军队院校中抽取 10 个教学班共计 513 名被试参 与问卷调查。发放调查问卷513份, 回收问卷486份, 剔除 无效问卷, 共得到有效调查问卷 426 份, 有效回收率为 $87.7 \%$ 。调查对象的人口学变量分布如下：男性 381 人 $(89.4 \%)$ ，女生 45 人 (10.6\%) ; 已婚 80 人 ( $18.8 \%)$, 未婚 346 人 $(81.2 \%)$; 本科学历 391 人 $(91.8 \%)$, 研究生 学历 35 人 $(8.2 \%)$; 有工作经历的 341 人 $(80.0 \%)$, 没有 工作经历的 85 人 $(20.0 \%)$; 年龄最小的 21 岁, 最大的 36 岁, 标准差为 3.03 岁。

\section{2. 研究工具}

\subsection{1. 主动性人格量表}

采用Bateman \& Crant开发的主动性人格量表进行测 量[11], 该量表也是测量个体主动性人格时的常用量表, 共有 17 项目、10项目、6项目、5 项目等多个版本，考虑到 被试作答时的心理状态及量表的信效度, 本研究选用 10 项目量表作为研究工具。该量表为单维结构, 采用Likert5 点计分法, 1-5（完全不符合-完全符合）, 得分越高, 表 明调查对象的主动性人格越强。本次调查中, 其Cronbach's $\alpha$ 系数为 0.799 。 


\subsection{2. 组织支持感量表}

Eisenberger编制的组织支持感量表 [17], 该量表也是 测量组织支持感时的常用量表, 具有较高的信效度, 单维 结构, 采用Likert5点计分, 1-5 (完全不符合-完全符合), 得分越高, 表明感知到的组织支持越强。本研究中, Cronbach's $\alpha$ 系数为 0.898 。

\subsection{3．工作旺盛感量表}

采用Porath等人开发的工作旺盛感量表[18], 共10个 题项, 分为学习和活力两个维度, 正式调查时将部分语句 进行了修改，如将“公司”改为“单位”，使之更加符合调查 对象的特点。该量表分为“活力”和“学习”两个维度, 其 Cronbach's $\alpha$ 系数分别为 0.816 和 0.799 , 总量表的 Cronbach's $\alpha$ 系数为 0.879 。

\section{3. 数据处理}

将研究对象的性别、年龄、婚姻状况、有无工作经历 等人口学变量作为额外变量, 在统计分析时进行控制。对 采集到的数据进行有效性检验, 剔除无效问卷并录入数据。 采用相关分析的方法, 探讨变量之间的共变关系, 采用回 归分析的方法, 探讨主动性人格对工作旺盛感的影响, 采 用结构方程模型分析的方法验证组织支持感在主动性人
格和工作旺盛感间的中介作用。使用的统计软件为 SPSS22.0和Lisrel8.70。

\section{3. 研究结果}

\section{1. 研究变量的区分效度检验}

采用验证性因素分析的方法对变量间的区分效度进 行检验, 结果如表1所示。三因子模型中各项拟合指标均 显著优于其他竞争模型, 且达到了最低的临界值标准。其 他竞争模型的拟合指标部分或者全部不达标, 由此说明, 预设的三因子模型与数据拟合良好, 研究中所使用的三个 变量分别代表三个不同的构念, 具有良好的区分度。

\section{2. 共同方法偏差检验}

由于本研究中收集到的数据全部来源了调查对象自 评, 不可避免会受到同源误差的影响, 因此, 本研究采用 Harman单因素法对可能存在的方法学效应进行检验。将调 查问卷中涉及到的所有题项进行因素分析, 未旋转因子分 析的结果显示: 共可以析出 4 个特征根大于 1 的公因子, 其 中第一公因子可解释总方差变异量的 $33.7 \%$, 低于 $40 \%$ 的 临界值标准。由此说明, 本研究不存在严重的共同方法偏 差, 研究结论具有较强的可信度。

表1 变量的区分效度检验。

\begin{tabular}{|c|c|c|c|c|c|c|c|}
\hline 模型 & $x^{2}$ & $d f$ & $\chi^{2} / d f$ & RMSEA & CFI & IFI & $\overline{\text { NNFI }}$ \\
\hline 三因子模型 & 484.4 & 132 & 3.7 & 0.079 & 0.95 & 0.95 & 0.94 \\
\hline 二因子模型a & 1940.8 & 134 & 14.5 & 0.179 & 0.85 & 0.85 & 0.83 \\
\hline 二因子模型b & 775.2 & 134 & 5.8 & 0.106 & 0.92 & 0.92 & 0.91 \\
\hline 单因子模型 & 2119.7 & 135 & 15.7 & 0.186 & 0.83 & 0.83 & 0.81 \\
\hline
\end{tabular}

注: 三因子模型为将每个变量视为独立变量; 二因子模型a将主动性人格和工作旺盛感合并为一个变量; 二因子模型b将主动性人格和组织支持感合 并为一个变量; 单因子模型为将三个变量合并为一个变量。

\section{3. 变量间的相关分析}

变量的均值、标准差及相关分析如表2所示, 人口学变 量与主变量的相关关系中, 除了性别与工作旺盛感显著正相 关外 $(r=0.10, p<0.05)$ ，其余相关均不显著；主变量间的
相关均达到了显著性水平, 其中主动性人格与组织支持感 $(r=0.33, p<0.01)$ 、工作旺盛感 $(r=0.40, p<0.01)$ 显著正 相关; 组织支持感与工作旺盛感显著正相关 $(r=0.52, p<0.01)$, 变量间的相关为后续研究假设的检验提供了基础。

表2 变量间的相关分析。

\begin{tabular}{|c|c|c|c|c|c|c|c|c|c|c|}
\hline 变量 & M & SD & 1 & 2 & 3 & 4 & 5 & 6 & 7 & 8 \\
\hline 1性别 & 1.11 & 0.31 & 1 & & & & & & & \\
\hline 2 学历 & 2.08 & 0.27 & $0.23^{* *}$ & 1 & & & & & & \\
\hline 3年龄 & 26.08 & 3.03 & $0.18^{* *}$ & $0.21^{* *}$ & 1 & & & & & \\
\hline 4婚否 & 1.81 & 0.39 & $-0.19^{* *}$ & $-0.12^{*}$ & $-0.55^{* *}$ & 1 & & & & \\
\hline 5工作经历 & 1.20 & 0.40 & -0.04 & 0.07 & $-0.42^{* *}$ & $0.21^{* *}$ & 1 & & & \\
\hline 6主动性人格 & 3.72 & 0.54 & -0.03 & 0.04 & -0.06 & 0.02 & -0.01 & 1 & & \\
\hline 7组织支持感 & 3.78 & 0.74 & 0.07 & 0.06 & 0.01 & -0.05 & 0.05 & $0.33^{* *}$ & 1 & \\
\hline 8工作旺盛感 & 4.20 & 0.60 & $0.10^{*}$ & 0.09 & 0.04 & 0.03 & -0.01 & $0.40^{* *}$ & $0.52^{* *}$ & 1 \\
\hline
\end{tabular}

注: "表示 $p<0.05,{ }^{* *}$ 表示 $p<0.01$.

\section{4. 组织支持感在主动性人格和工作旺盛感间的中介效 应检验}

采用结构方程模型的方法对组织支持感在主动性人 格和工作旺盛感间的中介作用进行检验。根据温忠麟等提
供的中介效应检验三步法, 首先做工作旺盛感对主动性人 格的回归, 结果显示, 主动性人格对工作旺盛感具有显著 的正向影响 $(\beta=0.60, p<0.001)$; 加入组织支持感的中介 作用后, 结构方程的拟合指数为 $\chi^{2} / \mathrm{df}=3.7, \mathrm{CFI}=0.95$, $\mathrm{IFI}=0.95, \mathrm{NNFI}=0.94, \mathrm{GFI}=0.89$, 此时主动性人格对组织 
支持感具有显著的正向预测作用 $(\beta=0.39, p<0.001)$, 组 织支持感对工作旺盛感的正向预测作用也显著 $(\beta=0.44$, $\mathrm{p}<0.001$ ), 主动性人格对工作旺盛感的直接效应仍然显
著 $(\beta=0.46, p<0.001)$, 如图2所示。由此证明, 组织支 持感在主动性人格和工作旺盛感间起部分中介作用, 中介 效应大小为 $0.39 \times 0.44=0.172$, 中介效应占总效应的 $27.2 \%$ 。

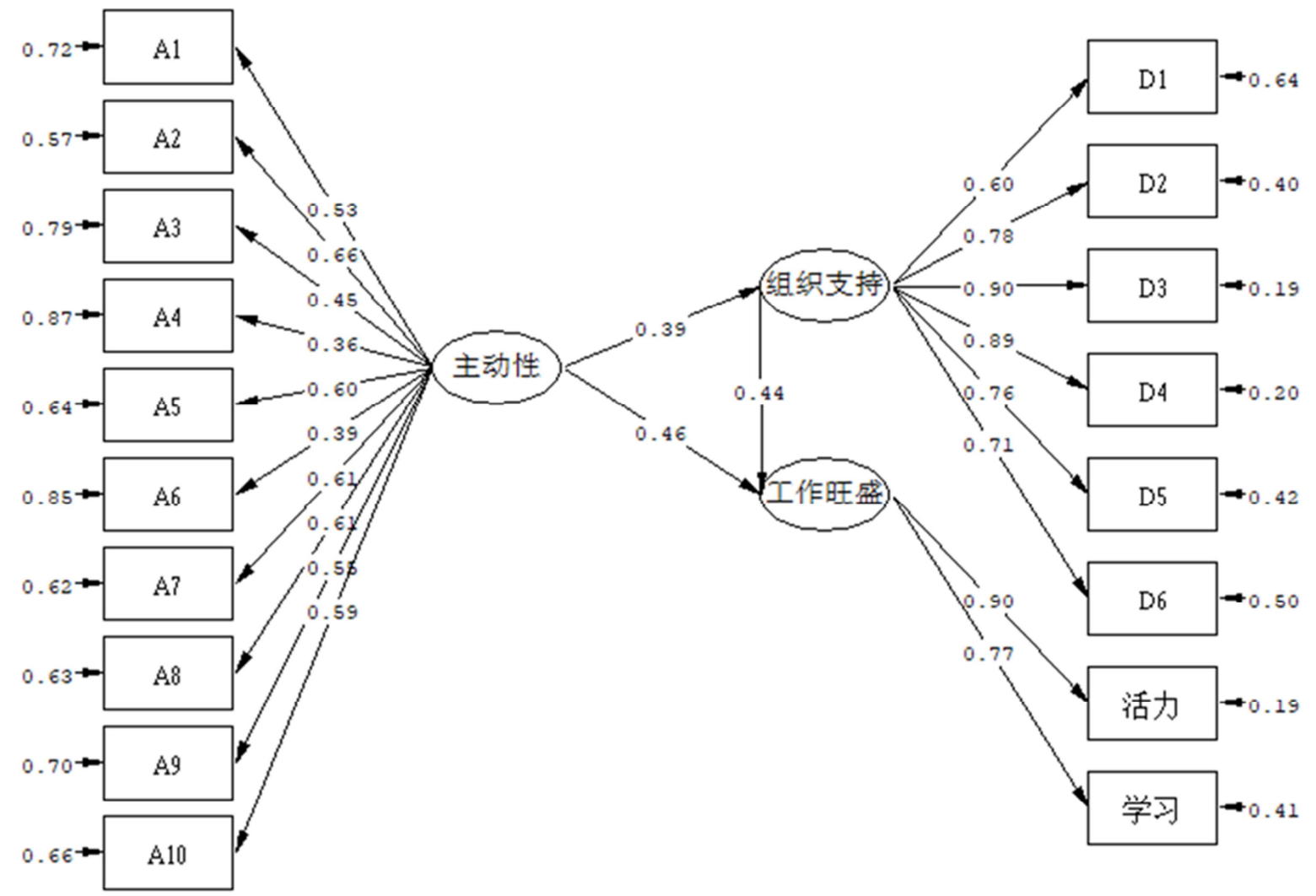

图2 组织支持感在主动性人格和工作旺盛感间的中介作用。

\section{4. 讨论}

\section{1. 文职人员主动性人格对工作旺盛感的影响}

研究结果显示, 文职人员主动型人格对工作旺盛感具 有显著的正向影响作用, 该结论与董昫华等人的研究结论 一致 [19]。一方面, 具有主动性人格的个体更容易适应环 境和参与学习, 更乐于将自己置身于可以感到工作旺盛感 的情境中 [20]; 另一方面, 主动性人格特质作为一种稳定 的人格特质, 同时也是一种内部动机, 这种内部动机必然 会对个体的工作行为产生影响, 促进文职人员在工作中表 现出更强的主动性, 积极探寻解决问题的不同思路和方法, 在面对工作中的挫折和困难时, 也往往能够采用更为积极 主动的行为应对, 而非被动放弃, 高主动性人格的个体对 待工作更加热情, 希望通过不断的学习以提高技能, 并会 投入更多的精力到工作中, 进而表现出更强的工作旺盛感。

\section{2. 组织支持感在文职人员主动性人格和工作旺盛感间 的中介作用}

本研究对主动性人格影响工作旺盛感的作用机制进 行了探讨, 结果表明, 文职人员主动性人格既可以直接对 工作旺盛感产生影响, 又可通过组织支持感的中介作用对 工作旺盛感产生间接影响, 也即组织支持感在主动型人格
和工作旺盛感间起“桥梁”作用, 部分传递了前者对后者的 促进作用。以往研究多倾向于将主动性人格和组织支持感 作为独立变量, 探讨两者间的交互作用 [21, 22], 而鲜有研 究探讨两者间的互依关系, 本研究则基于理论假设对其可 能存在的关系进行了验证, 证实了主动性人格对组织支持 感具有显著的正向影响。高主动性个体的目标感更强, 愿 意为了达成目标尝试各种方式来解决问题, 同时, 由于其 人际交往中主动性的特征, 组织内社会支持网络也更高效、 发达, 在平时的工作中尤其是遇到挫折性事件时, 能够获 取和利用的组织支持也更广泛有效。Cong等人的研究也表 明, 高主动性人格的个体更善于掌控当前自身所处的环境, 能够积极获取各种资源以实现自己的预定目标[23], 自然 会在工作中感知到更强的组织支持, 因此, 高主动性个体 的组织支持感更强。而组织支持感与工作旺盛感的关系在 多数研究中均已得到证实 [8], 组织支持感高的个体不仅能 在工作中得到更多的资源, 用于更好地完成现有工作, 维 持较高的工作绩效; 同时, 根据社会交换理论, 个体在工 作中一旦得到更多的组织支持, 在内心深处出于一种回报 组织的动机, 也更愿意表现出较好的工作状态, 尤其是在 中国这样一种注重“关系”和“人情”文化的社会环境中, 表 现得更为突出。因此, 组织支持感也会对工作旺盛感产生 显著的正向影响。基于以上分析可知, 组织支持感在文职 人员主动性人格和工作旺盛感间起中介作用。 


\section{3. 管理启示及研究局限}

本研究结论对军队文职人员管理也具有一定的启示, 对创新文职人员激励方法措施具有一定的指导。首先，鉴 于主动性人格对工作旺盛感的直接效应, 相关部门在进行 文职人员招聘时，同等条件下可优先考虑录用主动性人格 较强的候选对象, 对于已经招录或者现有文职人员, 可采 用一定的培训或训练等形式, 提高其行为的主动性; 其次, 组织也要立足实际, 以人为本, 创设良好的组织支持氛围, 在文职人员遇到工作或生活中的困难时, 给与及时有效的 帮助, 只有使其感受到组织的温暖, 才能使其在工作中充 分投入，体会到更强的工作旺盛感。

然而, 本研究也有一些设计上的缺陷, 可在后续研究 中改进。首先, 本研究所用数据全部为同一时间点的自评 形式所获得, 在揭示变量间因果关系方面具有天然的缺陷, 后续研究可考虑采用纵向研究或追踪研究的方式, 更为准 确地验证变量间的因果关系; 其次, 本研究仅仅探索了组 织支持感单一变量的中介效应, 实际过程中的因果关系可 能更为复杂, 可能存在多个中介变量或调节变量同时对两 者间关系产生影响的情况, 后续研究可对此进行研究, 以 更全面地揭示主动性人格影响工作旺盛感的内部机制和 边界条件。

\section{5. 结论}

本研究以问卷调查的形式, 通过相关分析、结构方程 建模等统计方法, 探讨了军队文职人员主动性人格对工作 旺盛感的影响及内部作用机制。结果发现, 军队文职人员 主动性人格、组织支持感、工作旺盛感间均存在显著的正 相关关系, 主动性人格得分越高, 组织支持感和工作旺盛 感的得分相应越高。结构方程模型检验的结果显示, 主动 性人格对工作旺盛感具有显著的促进作用, 组织支持感在 主动性人格和工作旺盛感间起部分中介作用, 主动性人格 除了对工作旺盛感产生直接影响外, 还可通过影响组织支 持感进而影响工作旺盛感。

本研究对军队文职人员的管理和激励也具有一定的 启示，提高军队文职人员的工作旺盛感可以从招聘、培训 等环节入手，选拔或塑造其主动性人格，同时，也要加大 对文职人员的组织支持力度, 使其感受到更强的组织支持 感。

\section{致谢}

本文为国家社科基金项目《新时代军队文职人员思想 政治素质研究》（19XGL028）和《新时代军队文职人员 职业精神研究》（20BGL312）的阶段性成果之一。

\section{参考文献}

[1] Spreitzer G, Sutcliffe K, Dutton J, et al. A Socially Embedded Model of Thriving at Work[J]. Organization Science, 2005, 16 (5): 537-549.
[2] Jiang Z. Proactive personality and career adaptability: The role of thriving at work [J]. Journal of Vocational Behavior, 2017, 98: 85-97.

[3] Kristin H, A S C, Carmen B. Transformational leadership and burnout: The role of thriving and followers' openness to experience. [J]. Journal of occupational health psychology, 2018, 23 (1): 31-43.

[4] Walumbwa F O, Muchiri M K, Misati E, et al. Inspired to perform: A multilevel investigation of antecedents and consequences of thriving at work[J]. Journal of Organizational Behavior, 2018, 39 (3): 249-261.

[5] Spreitzer G, Porath C L, Gibson C B. Toward human sustainability: How to enable more thriving at work $[\mathrm{J}]$. Organizational Dynamics, 2012, 41 (2): 155-162.

[6] 安静, 万文海. 诚信领导对员工工作繁荣作用的实证研究 一心理安全感的中介作用 [J]. 科技与经济, 2014, 27 (05): 75-79.

[7] 陈洪安, 李乐, 刘俊红, 等. 职场友谊对员工工作繁荣影响 的实证研究 [J]. 华东师范大学学报(哲学社会科学版), 2016, 48 (05): 150-160.

[8] 时勘，万金，崔有波. 基于人-情境交互作用的工作旺盛感 生成机制[J]. 中国人力资源开发, 2015(17):65-72.

[9] 陶厚永, 韩玲玲, 章娟. 何以达到工作旺盛?工作支持与家 庭支持的增益作用 $[\mathrm{J}]$. 中国人力资源开发，2019，36 (03): 117-132.

[10] 刘丽华, 黎思源. “90后”工作旺盛感的形成机制研究——基 于组织社会化视角 [J]. 软科学, 2020, 34 (08): 103-108.

[11] Bateman T S, Crant J M. The Proactive Component of Organizational Behavior: A Measure and Correlates[J]. Journal of Organizational Behavior, 1993, 14 (2): 103-118.

[12] 周愉凡, 张建卫, 张晨宇, 等. 主动性人格对研发人员创新 行为的作用机理一一基于特质激活与资源保存理论整合性 视角 $[J]$. 软科学, 2020, 34 (07): 33-37.

[13] 刘春莉, 江琦, 刘佳凝, 等. 变革型领导对进谏行为的影响: 主动性人格和正性情绪、负性情绪的作用 [J]. 西南大学学 报(自然科学版), 2020, 42 (06): 100-109.

[14] 叶宝娟, 孙原, 高良, 等. 主动性人格与大学生职业成熟度 的关系:一个有调节的中介模型 $[\mathrm{J}]$. 心理发展与教育, 2020, 36 (03): 304-310.

[15] 凌文轻, 杨海军, 方俐洛. 企业员工的组织支持感 [J]. 心理 学报, 2006 (02): 281-287.

[16] 王姣艳，郝晓川，李扬. 组织支持与特殊教育教师职业幸 福感的关系: 链式中介效应分析 [J]. 中国临床心理学杂志, 2020 (06): 1281-1284.

[17] Eisenberger R, Huntington R, Hutchison S, et al. Perceived Organizational Support [J]. Journal of Applied Psychology, 1986, 71 (3): 500-507.

[18] Porath C, Spreitzer G, Gibson C, et al. Thriving at work: Toward its measurement, construct validation, and theoretical refinement $[\mathrm{J}]$. Journal of Organizational Behavior, 2012, 32 (2): $250-275$. 
[19] 董昫华，童卉. 高职教师工作资源对工作旺盛感的影响:个 人主动性和主动性人格的多重中介效应 $[\mathrm{J}]$. 江苏高职教育, 2019, 19 (02): 69-74.

[20] Crant, Michael J. The Proactive Personality Scale and Objective Job Performance Among Real Estate Agents [J]. Journal of Applied Psychology, 1995, 80 (4): 532-537.

[21] 张宁. 组织社会化策略、组织支持感对90后员工离职倾向 影响的实证研究[D]. 山东大学, 2018.
[22] 陈可. 主动性人格对员工建言行为的影响研究[D]. 南京师 范大学, 2014.

[23] Gong Y, Cheung S Y, Wang M, et al. Unfolding the Proactive Process for Creativity: Integration of the Employee Proactivity, Information Exchange, and Psychological Safety Perspectives [J]. Journal of Management, 2012, 36 (5): 603-612. 